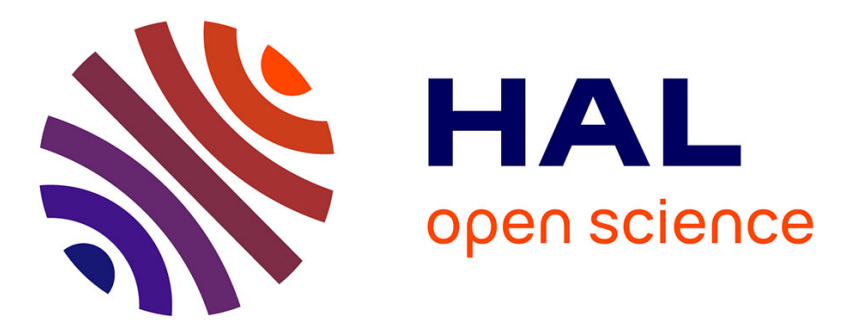

\title{
Spin relaxation time of donor-bound electrons in a CdTe quantum well
}

\author{
G. Garcia-Arellano, Frédéric Bernardot, G. Karczewski, C. Testelin, M.
}

Chamarro

\section{- To cite this version:}

G. Garcia-Arellano, Frédéric Bernardot, G. Karczewski, C. Testelin, M. Chamarro. Spin relaxation time of donor-bound electrons in a CdTe quantum well. Physical Review B: Condensed Matter and Materials Physics (1998-2015), 2019, 99 (23), pp.202202. 10.1103/PhysRevB.99.235301 . hal02390027

\section{HAL Id: hal-02390027 \\ https://hal.science/hal-02390027}

Submitted on 14 Jul 2020

HAL is a multi-disciplinary open access archive for the deposit and dissemination of scientific research documents, whether they are published or not. The documents may come from teaching and research institutions in France or abroad, or from public or private research centers.
L'archive ouverte pluridisciplinaire HAL, est destinée au dépôt et à la diffusion de documents scientifiques de niveau recherche, publiés ou non, émanant des établissements d'enseignement et de recherche français ou étrangers, des laboratoires publics ou privés. 


\title{
Spin relaxation time of donor-bound electrons in a CdTe quantum well
}

\author{
G. Garcia-Arellano, ${ }^{1}$ F. Bernardot, ${ }^{1}$ G. Karczewski, ${ }^{2}$ C. Testelin, ${ }^{1}$ and M. Chamarro ${ }^{1}$ \\ ${ }^{1}$ Sorbonne Université, CNRS, Institut des NanoSciences de Paris, 4 Place Jussieu, F-75005 Paris, France \\ ${ }^{2}$ Institute of Physics, Polish Academy of Sciences, Al. Lotników 32/46, PL-02668 Warsaw, Poland
}

(Received 7 February 2019; revised manuscript received 18 April 2019; published 4 June 2019)

\begin{abstract}
Low-temperature spin relaxation time of electrons localized on iodine donors placed in the middle of a 8-nm CdTe quantum well is studied by using the photoinduced Faraday rotation technique, for donor concentrations in the range from $1 \times 10^{9} \mathrm{~cm}^{-2}$ to $3.6 \times 10^{11} \mathrm{~cm}^{-2}$. A maximum relaxation time of the order of $20 \mathrm{~ns}$ is found at a doping concentration near the metal-insulator transition. Adapting the theoretical description developed for bulk GaAs [R. I. Dzhioev et al., Phys. Rev. B 66, 245204 (2002)] to a quantum well, we have fitted the experimental results taking as a parameter the spin-orbit constant in CdTe. A satisfactory fit is obtained with $\alpha_{s o}=0.079 \pm 0.011$, which is also very close to the calculated theoretical value. A comparison of the experimental spin relaxation times measured previously in bulk CdTe to those predicted by the theory is done. Finally, we compare the predicted spin relaxation times in bulk CdTe to those predicted in one of the most studied bulk semiconductor materials: GaAs, and in (less studied) $\mathrm{ZnSe}$.
\end{abstract}

DOI: 10.1103/PhysRevB.99.235301

\section{INTRODUCTION}

Dopants in semiconductor devices control their electric, optical or magnetic properties. The inevitable device downscaling brings dopant impurities in the foreground, not only for classical electronics, but also for quantum information and communication [1,2]. Indeed, the shallow paramagnetic impurities are promising candidates for basic units of the quantum computers, i.e., for qubits, due to their easy scalability and homogeneity. In recent years, a large number of studies has been centered on impurities in silicon showing long spin lifetimes [3,4], but due to an indirect band gap, these impurities cannot be optically addressed. In contrast, the direct-band-gap semiconductors exhibit efficient optical transitions. Impurities in GaAs have been largely studied in the past. In 1977 Weisbuch reported experimental studies on spin relaxation of electrons localized on donors or acceptors [5]. They showed up spin relaxation times up to $30 \mathrm{~ns}$ in lightly doped $n$-type crystals of GaAs. Subsequent measurements made by Kikkawa and Awschalom [6] reported times around $130 \mathrm{~ns}$ at $n_{d}=10^{16} \mathrm{~cm}^{-3}$. All these results motivated intensive research on the influence of doping on spin relaxation times. In bulk GaAs, a complete study of the doping concentration influence on the relaxation time of localized electrons was performed by Dzhioev et al. [7] and recently revisited by Belykh et al. [8]. In Ref. [7], the spin relaxation times were measured using the Hanle effect with $n_{d}$ spanning the range from $10^{14}$ to $10^{17} \mathrm{~cm}^{-3}$, whereas in Ref. [8] pumpprobe experiments were used for a similar study. Recently, the spin-noise spectroscopy performed in bulk $n$-GaAs samples at low temperatures and with concentrations in the insulating regime, resulted in smaller spin relaxation times than those obtained by Hanle measurements [9]. A detailed comparison of the Hanle experiment and spin-noise spectroscopies has been performed by Crooker et al. [10].

The influence of the doping concentration on the spin relaxation time has been studied at low temperature in other bulk materials such as $\mathrm{ZnO}$ [11], InSb [12], InAs [13], GaN [14,15], and $\mathrm{ZnSe}$ [16], for different doping values in the insulating regime and beyond the metal-insulator transition. Recently, a study in the insulating regime was performed in bulk CdTe by Sprinzl et al. [17], reporting a nonmonotonous behavior exhibiting a maximum value of the spin relaxation time of $2.5 \mathrm{~ns}$ at $n_{d}=4.9 \times 10^{16} \mathrm{~cm}^{-3}$ and a minimum value of $40 \mathrm{ps}$ at $n_{d}=1.5 \times 10^{13} \mathrm{~cm}^{-3}$.

From the theoretical point of view, the influence of doping on the spin relaxation time at low temperature has been explained as the interplay of different relaxation mechanisms: in the insulating regime and at high doping concentration, the anisotropic exchange interaction mechanism dominates, while at low doping density, the hyperfine nuclear interaction is the most important mechanism $[7,18,19]$. In the metallic regime, the spin relaxation is dominated by the D'yakonov-Perel mechanism: the spin lifetime reaches a second maximum near the metal-insulator transition, and at higher doping concentrations, the spin relaxation time decreases with concentration.

In order to avoid other relaxation mechanisms appearing in wurtzite materials, such as $\mathrm{GaN}$ or $\mathrm{ZnO}$ [20], and make comparisons to one of the most studied systems, bulk GaAs, we have centered our study on CdTe. This compound crystalizes in the same crystal structure as GaAs, however the spin-orbit interaction is higher than in GaAs, and other parameters that determine the spin relaxation time are very different from those of GaAs (effective mass, electron Landé factor, Bohr radius, etc.). In this article, we present a study of the spin relaxation time dependence on the concentration of electrons localized on donors located in the middle of a 8-nm CdTe quantum well (QW). In this kind of nanostructure, the localization of the electron wave function is significantly enhanced with respect to three-dimensional (3D) systems [21-24]. Moreover, QWs are also known to purify the optical selection rules for circularly polarized light, and then allow one to obtain a higher optical orientation degree of the 
electron spins than in 3D materials. We found a relaxation time approximately 10 times larger than the maximum relaxation time reported in bulk CdTe in Ref. [17]. By adapting the bulk theoretical description of two main mechanisms playing a dominant role in the spin relaxation times of electrons bound to donors in the insulating regime at low temperature, in the case of a $\mathrm{QW}$, we estimated from experimental data the value for the dimensionless spin-orbit constant in CdTe: $\alpha_{s o}=$ $0.079 \pm 0.011$. We calculated the electronic spin relaxation time in bulk CdTe to compare it with available experimental results [17], which are at least one order of magnitude smaller than the spin relaxation time found in our QW system and two orders of magnitude smaller than those predicted by the theory for a bulk system. We showed that the theoretical spin relaxation times in bulk CdTe are larger than in QWs, when the same mechanisms are considered. We then discuss the possible sources of additional spin relaxation mechanisms that can be present during experimental measurements. Finally, we discuss and compare theoretical and experimental results in a very well-studied semiconductor, GaAs, to another less studied bulk semiconductor, ZnSe. From the theoretical calculation developed here, this II-VI material appears to be a good candidate for observation of long electron spin relaxation times, of the order of $\mu \mathrm{s}$.

\section{SAMPLES AND PHOTOLUMINESCENCE CHARACTERIZATION}

Each of the six studied samples consist of a 8-nm thick CdTe QW confined between CdMgTe barriers grown by molecular beam epitaxy on a (100)-oriented GaAs substrate. The samples were doped with iodine donors placed in the central plane of the QW, with different nominal concentrations: (A) undoped, (B) $3.2 \times 10^{10} \mathrm{~cm}^{-2}$, (C) $9.7 \times 10^{10} \mathrm{~cm}^{-2}$, (D) $1.6 \times 10^{11} \mathrm{~cm}^{-2}$ (E) $2.9 \times 10^{11} \mathrm{~cm}^{-2}$, and (F) $3.6 \times$ $10^{11} \mathrm{~cm}^{-2}$.

Figure 1 shows the photoluminescence (PL) spectra of the samples $\mathrm{A}, \mathrm{C}$, and $\mathrm{E}$, obtained after excitation with a 5-mW 633-nm He-Ne laser, at the temperature $T=10 \mathrm{~K}$. The free-exciton emission at $1.625 \mathrm{eV}(X)$ dominates the PL spectrum of the intentionally undoped sample $\mathrm{A}$, as is shown in Fig. 1(a). A residual concentration of donors in this sample is revealed by a second peak at $1.621 \mathrm{eV}$, associated with the donor-bound exciton transition $\left(D^{0} X\right)$ (see Kheng et al. [25]). The energy difference between the free-exciton peak and the $D^{0} X$ one gives the binding energy $\approx 4 \mathrm{meV}$ to form the $D^{0} X$ complex. The shoulder at lower energy is associated with the formation of excitons bound to neutral acceptors $\left(A^{0} X\right)$ [26]; indeed, the introduction of donor impurities in the $\mathrm{QW}$ creates compensation sites, inducing the presence of acceptor sites, which in our case are probably cadmium vacancies. For the intermediate doping concentration, sample C [see Fig. 1(b)], a shoulder associated with the exciton transition at $1.615 \mathrm{eV}$ is still visible in the high-energy part of the $D^{0} X$ broad band. At higher donor concentration, only a broad peak associated with the $D^{0} X$ transition is visible [see Fig. 1(c)].

The full width at half maximum of the $D^{0} X$ PL band increases with the increasing donor concentration, from $2.6 \mathrm{meV}$ for sample A to $6.2 \mathrm{meV}$ for sample E. The exciton PL band also broadens, weakens, and finally vanishes
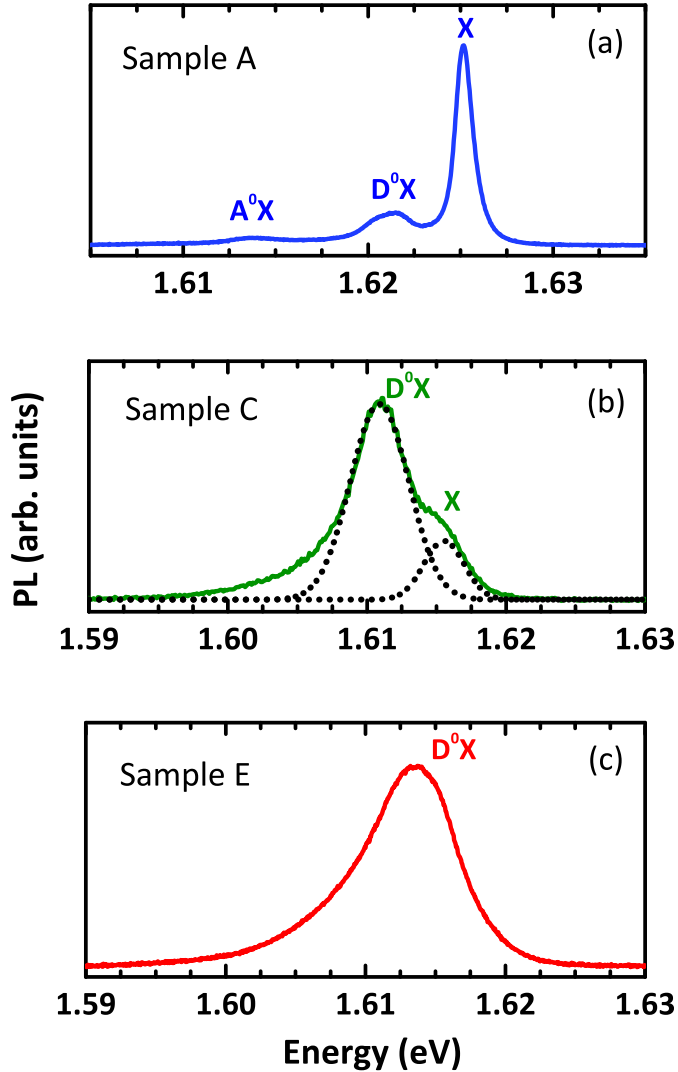

FIG. 1. PL spectra, at $2 \mathrm{~K}$, of 8-nm CdTe QWs containing in their center planes, different donor concentrations: (a) sample A: $1 \times 10^{9} \mathrm{~cm}^{-2}$, (b) sample C: $9.7 \times 10^{10} \mathrm{~cm}^{-2}$, and (c) sample E: $2.9 \times 10^{11} \mathrm{~cm}^{-2}$.

when the concentration of donor increases. When comparing Figs. 1(a) and 1(b), we underline that the energy of the $X$ transition is slightly different; this is because of the $\mathrm{Mg}$ content in the barrier: for sample $\mathrm{A}$, the $\mathrm{Mg}$ content is $18 \%$, while it is $11 \%$ for sample $\mathrm{C}$ (and also for the other samples).

\section{SPIN DYNAMICS MEASUREMENTS}

To study the spin dynamics of resident electrons in our sample, we used the photoinduced Faraday rotation (PFR) technique. The light source is a Ti:Sapphire laser beam with a 2-ps pulse duration and a 76- $\mathrm{MHz}$ repetition rate, which is split into pump and probe beams. The pump beam is $\sigma+/ \sigma-$ modulated at $500 \mathrm{kHz}$ with an electro-optic modulator, in order to avoid nuclear spin polarization by the optical orientation of the electrons. The probe beam is linearly polarized, and its intensity is modulated with an acousto-optic modulator at 3 $\mathrm{kHz}$. The rotation angle of the probe beam polarization is analyzed after transmission by an optical bridge. A double lock-in amplifier is used in order to improve the signal-to-noise ratio. After removal of its GaAs substrate, the studied sample is placed in a liquid-helium cryostat. A superconducting split coil immerged in the cryostat is used to apply a magnetic field in Voigt geometry (in the plane of the sample, and perpendicular to the light propagation direction).

Figure 2 shows the PFR signal obtained at low temperature, $T=2 \mathrm{~K}$, in the presence of a transverse magnetic field 


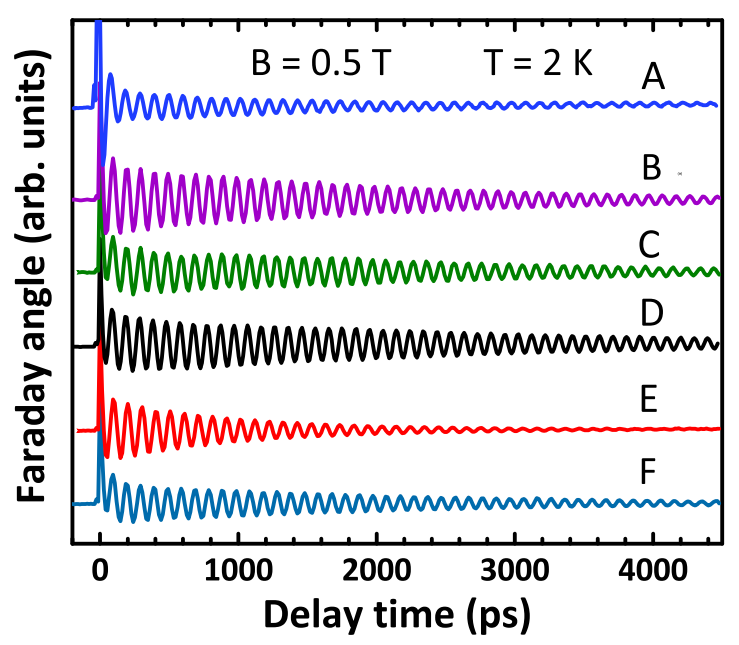

FIG. 2. PFR signals as a function of the pump-probe delay for samples A-F. The PFR signals were obtained at $T=2 \mathrm{~K}, B=0.5 \mathrm{~T}$, using the following optical energies: (A) $1.622 \mathrm{eV}$, (B) $1.613 \mathrm{eV}$, (C) $1.611 \mathrm{eV}$, (D) $1.615 \mathrm{eV}$, (E) $1.617 \mathrm{eV}$, and (F) $1.612 \mathrm{eV}$.

of $0.5 \mathrm{~T}$ for the six studied samples. The common pump and probe energy is always tuned to the $D^{0} X$ transition in order to create mainly donor-bound exciton complexes, and to probe the $D^{0} X$ optical transition, since the band-width of the used mode-locked Ti:Sapphire laser is less than $1 \mathrm{meV}$. The focus spot area is $(90 \mu \mathrm{m})^{2}$ and the energy fluence of the pump pulses is $0.09 \mu \mathrm{J} \mathrm{cm}^{-2}$. Figure 2 shows that for all the samples, an oscillatory signal with an envelope time larger than the lifetime of the $D^{0} X$ complex $T_{\mathrm{R}}$ ( $\sim 200$ ps Ref. [26]) is observed. This long-lasting signal is the signature of the spin polarization of electrons bound to donors $D^{0}$. The mechanism involved in the creation of this electron polarization has been discussed in Ref. [23].

When a transverse magnetic field $B$ is applied in the QW plane, the Larmor frequency associated to the carriers is

$$
\Omega_{e, h}=\frac{g_{e, h}^{\perp} \mu_{B}}{\hbar} B,
$$

where $\mu_{B}$ is the Bohr magneton, $\hbar$ is the reduced Planck constant, and $g_{e, h}^{\perp}$ is the transverse electron (hole) Landé factor. For holes the Landé factor $g_{h}^{\perp}$ is almost zero in a 8-nm CdTe QW [27], so the field induces a spin precession of the resident electrons bound to the donors, but not of the $D^{0} X$ complex since the $D^{0} X$ complex contributes to the spin dynamics by its hole.

The electronic spin dynamics of $D^{0}$ is described by the equation [23]

$$
\frac{d \vec{S}_{\perp}}{d t}=\vec{\Omega}_{e} \wedge \vec{S}_{\perp}-\frac{\vec{S}_{\perp}}{T_{2}^{*}}+\frac{J(t)}{T_{R}} \vec{e}_{z}
$$

where $\vec{S}_{\perp}$ is the transverse component (to the magnetic field) of the electronic spin, $\vec{\Omega}_{e}$ is the Larmor precession vector of the electrons, $T_{2}^{*}$ is the spin decoherence time of the electronic spins, and $J(t)=J_{0} e^{-t / \tau}$ is one third of the average kinetic momentum of the ensemble of $D^{0} X$ complexes; $\tau$ is the decay time of the difference between the $D^{0} X_{+3 / 2}$ and $D^{0} X_{-3 / 2}$ populations.
The solution of Eq. (2), for times much larger than $\tau$, is

$$
S_{z}(t)=A_{2} e^{-t / T_{2}^{*}} \cos \left(\Omega_{e} t\right),
$$

but for times of the order of $\tau$, the next expression fits the PFR signal:

$$
J_{z}(t)+S_{z}(t)=A_{1} e^{-t / \tau}+A_{2} e^{-t / T_{2}^{*}} \cos \left(\Omega_{e} t\right) .
$$

The first nonoscillatory component is clearly seen in Fig. 2, especially in samples with high concentrations of donors (see Fig. 2, curves C-F). However, when the sample contains a low concentration of donors it is possible to observe another additional oscillatory component of the PFR signal, having a frequency similar to the electron one and with a very short damping time. This additional component corresponds to the oriented exciton spins (see Fig. 2, curve A).

Figure 3(a) shows the PFR signal at different magnetic fields for sample D. For low magnetic fields, the oscillatory behavior of the PFR signal is also observed at negative delays. This means that the damping time of the oscillations is comparable to the repetition period $13.2 \mathrm{~ns}$ of the laser, and this damping time decreases for increasing magnetic fields.

We have fitted expression (4) to all the PFR curves. Figure 3(b) shows the dependence of $\Omega_{e}$ on the applied magnetic field. A linear fit of the data gives $g_{e}^{\perp}=1.45$. In Fig. 3(b) we present the other fitting parameter, $1 / T_{2}^{*}$, as a function of the magnetic field, which is proportional to the magnetic field due to the inhomogeneities of the $g_{e}^{\perp}$ values, as it is shown below. Assuming a Lorentzian distribution of $g_{e}^{\perp}$ factors, the experimentally determined $T_{2}^{*}$ is related to the zero-field decoherence time $T_{2}(0)$ as follows:

$$
\frac{1}{T_{2}^{*}}=\frac{1}{T_{2}(0)}+\Delta g_{e} \frac{\mu_{B}}{\hbar} B
$$

From a linear fit to the expression (5) of the spin dephasing rates $1 / T_{2}^{*}$, shown in Fig. 3(b), we obtain the corresponding spin decoherence time $T_{2}(0)$ for each sample. Note that, when we extrapolate $T_{2}^{*}$ to zero magnetic field, we expect $T_{2}^{*}(0)=$ $T_{2}(0)=T_{1}$ where $T_{1}$ is the longitudinal spin relaxation time. Figure 3(c) shows the fit of Eq. (4) to the PFR signal at $B=0.5 \mathrm{~T}$. The characteristic time, $\tau$, of the $D^{0} X$ complex is equal to $60 \mathrm{ps}$ and does not change very much as a function of magnetic field or doping concentration. This time contains two contributions: the recombination rate of $D^{0} X$ and the hole spin flip rate in the $D^{0} X$ state. The lifetime of $D^{0} X$ has been measured in a previous publication for a sample containing approximately $10^{11} \mathrm{~cm}^{-2}$ [26]. We have no information about the evolution of $D^{0} X$ lifetime with the doping concentration, so we cannot draw a conclusion about the spin flip rate dependence on the doping concentration of the $D^{0} X$ complex. In the following we denote $T_{2}(0)=\tau_{s}$.

Figures 4(a) and 4(b) show the PFR signals for different magnetic fields and for the samples $\mathrm{E}$ and $\mathrm{F}$, respectively. Both samples $\mathrm{E}$ and $\mathrm{F}$ are near the metal-insulator transition. The metal-insulator transition in 3D materials has been calculated to appear for donor distances around three times the Bohr radius [28]; with this criterion, samples A-E are in the insulator regime, and sample $\mathrm{F}$ is in the metallic regime. We underline that the PFR signals are longer lasting for sample $F$ than for sample $\mathrm{E}$, and as a consequence, we find a $\tau_{s}$ much 

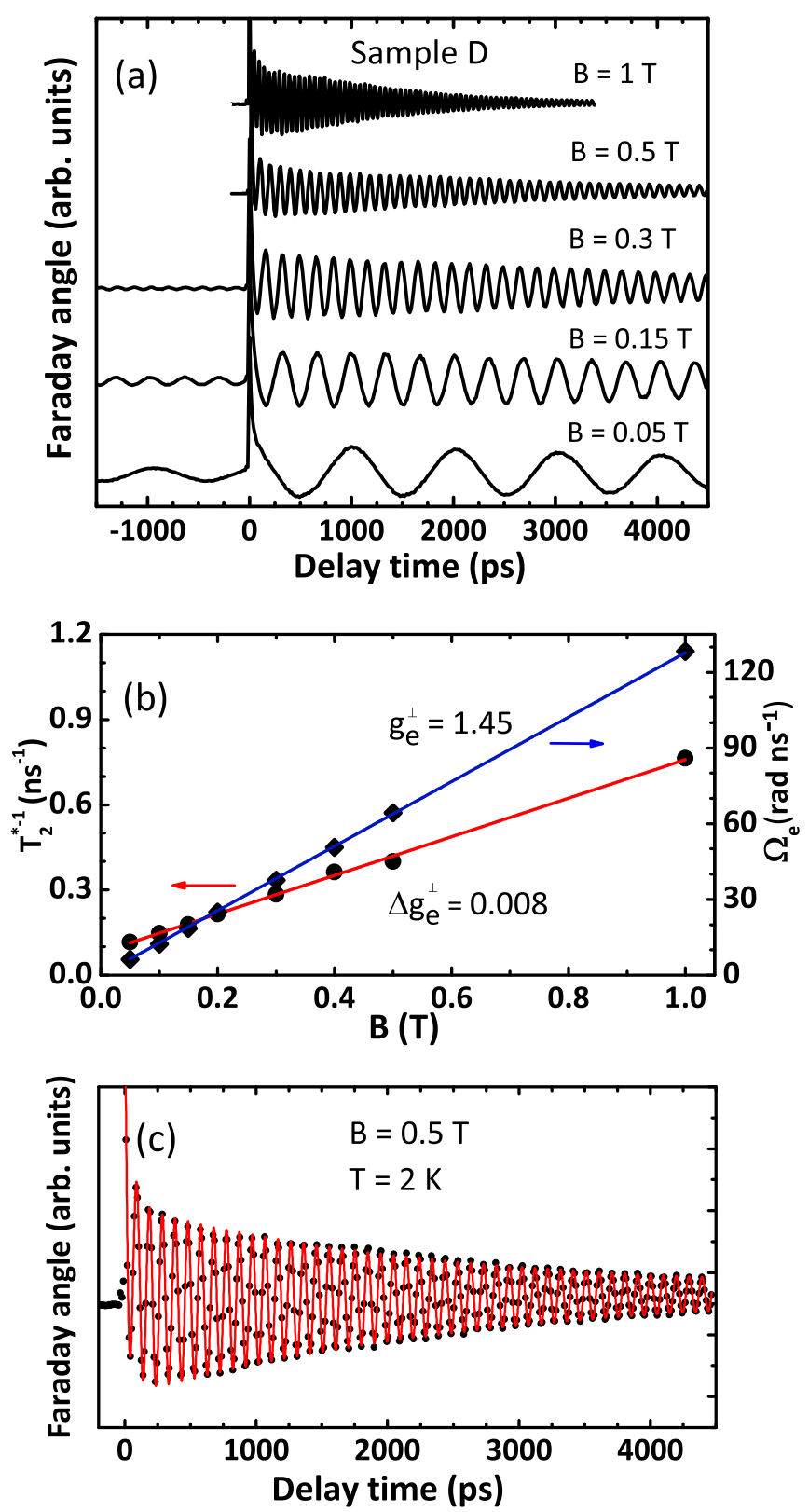

FIG. 3. (a) PFR signals for sample D as a function of the pumpprobe delay, obtained at $T=2 \mathrm{~K}$ for several values of the transverse magnetic field. The PFR curves are vertically shifted for clarity. (b) Experimental data of the $D^{0}$ Larmor frequency (diamonds) and the inverse of the spin decoherence time $T_{2}^{*}$ (circles). A linear fit according to Eq. (5) leads to $T_{2}(0)=12.5 \pm 0.8 \mathrm{~ns}$ and $\Delta g_{e}^{\perp}=0.0077 \pm$ 0.0002. A linear fit to Eq. (1) leads to the value $g_{e}^{\perp}=1.45$. (c) PFR signal (black points) obtained at $B=0.5 \mathrm{~T}$ and $T=2 \mathrm{~K}$. The red solid line is the fit of the expression: $0.08 \exp (-t / 20) \cos (0.06 t)+$ $0.031 \exp (-t / 2550) \cos (0.06 t))+0.04 \exp (-t / 60)$ to the experimental data, $t$ in picoseconds; only two terms are analyzed in Sec. III, the first oscillatory term corresponds to the exciton contribution.

longer for sample $\mathrm{F}$ than for sample $\mathrm{E}$. This peculiarity was also observed in bulk GaAs [7].

Figure 5(a) shows the spin relaxation time measured in samples A-F (full disks) and in one sample with the same characteristics and studied by using the same technique in Ref. [23], with a donor concentration of $1.2 \times 10^{11} \mathrm{~cm}^{-2}$

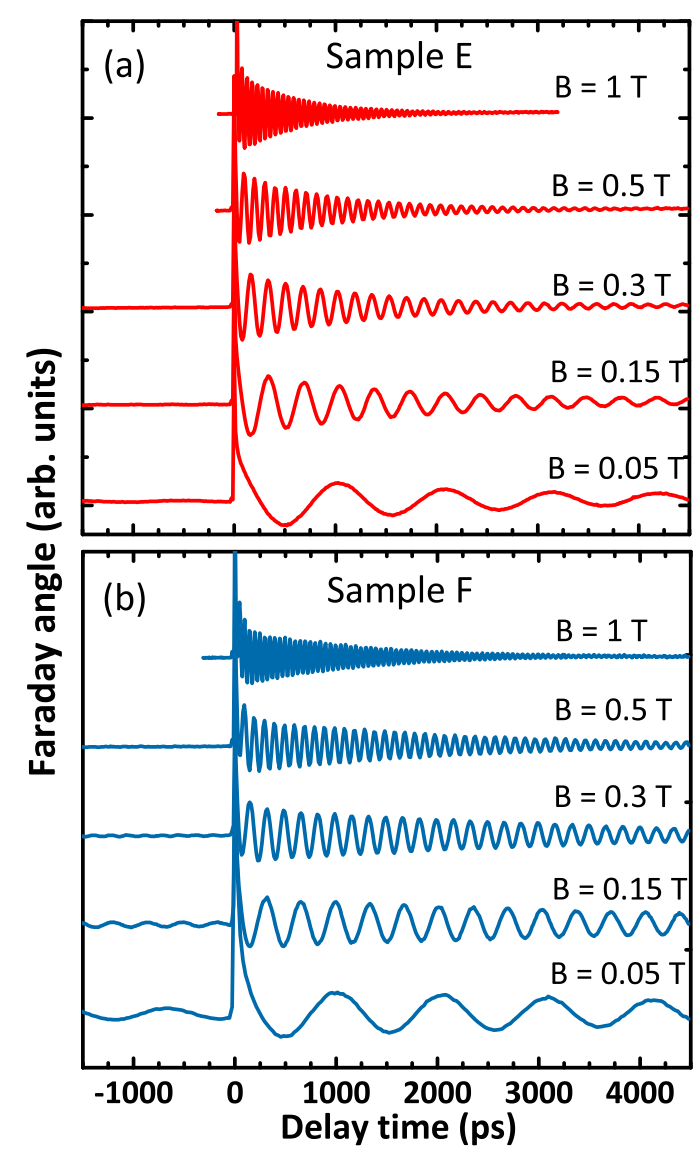

FIG. 4. PFR signals measured at $2 \mathrm{~K}$ in (a) sample $\mathrm{E}$ and (b) sample $\mathrm{F}$, as a function of the pump-probe delay for several values of the transverse magnetic field. The curves are shifted for clarity.

(full diamond). The spin relaxation time versus concentration shows a nonmonotonous behavior similar to the one found in bulk GaAs [7] and later in bulk CdTe [17]. The most remarkable feature of the dependence is a pronounced maximum of the relaxation time $(\sim 20 \mathrm{~ns})$ at a donor concentration of about $1.2 \times 10^{11} \mathrm{~cm}^{-2}$. In bulk CdTe the maximum $(2.5 \mathrm{~ns})$ appears at the concentration of $5 \times 10^{16} \mathrm{~cm}^{-3}$ [17], which corresponds approximately to a surface concentration of $1.4 \times$ $10^{11} \mathrm{~cm}^{-2}$. We also notice that for low doping concentrations in bulk CdTe, $1.5 \times 10^{13} \mathrm{~cm}^{-3}\left(\sim 1 \times 10^{9} \mathrm{~cm}^{-2}\right)$, Ref. [17] reported $\tau_{s}=40 \mathrm{ps}$, which is 100 times smaller than the spin relaxation time we measured in a 8-nm QW (4.5 ns). We also underline that, as it was remarked in the study carried out by Dzhioev et al. [7] in bulk GaAs, after the metalinsulator transition, another maximum of the spin relaxation time appears. We have observed the same phenomenon with sample $\mathrm{F}$, for which $\tau_{s}=14.4 \mathrm{~ns}$; this spin relaxation time is larger than $\tau_{s}=4.1 \mathrm{~ns}$ measured in sample E.

Figure 5(b) shows the dependence of the $g_{e}^{\perp}$ values on the doping concentration. We observe a similar dependence as the one shown by the spin relaxation time. This has been already noticed in bulk CdTe [17], and it is an indication of a strong correlation of the $g_{e}^{\perp}$ values with the spin relaxation mechanisms. But the reason in not clear yet, and more studies are needed in this subject. 

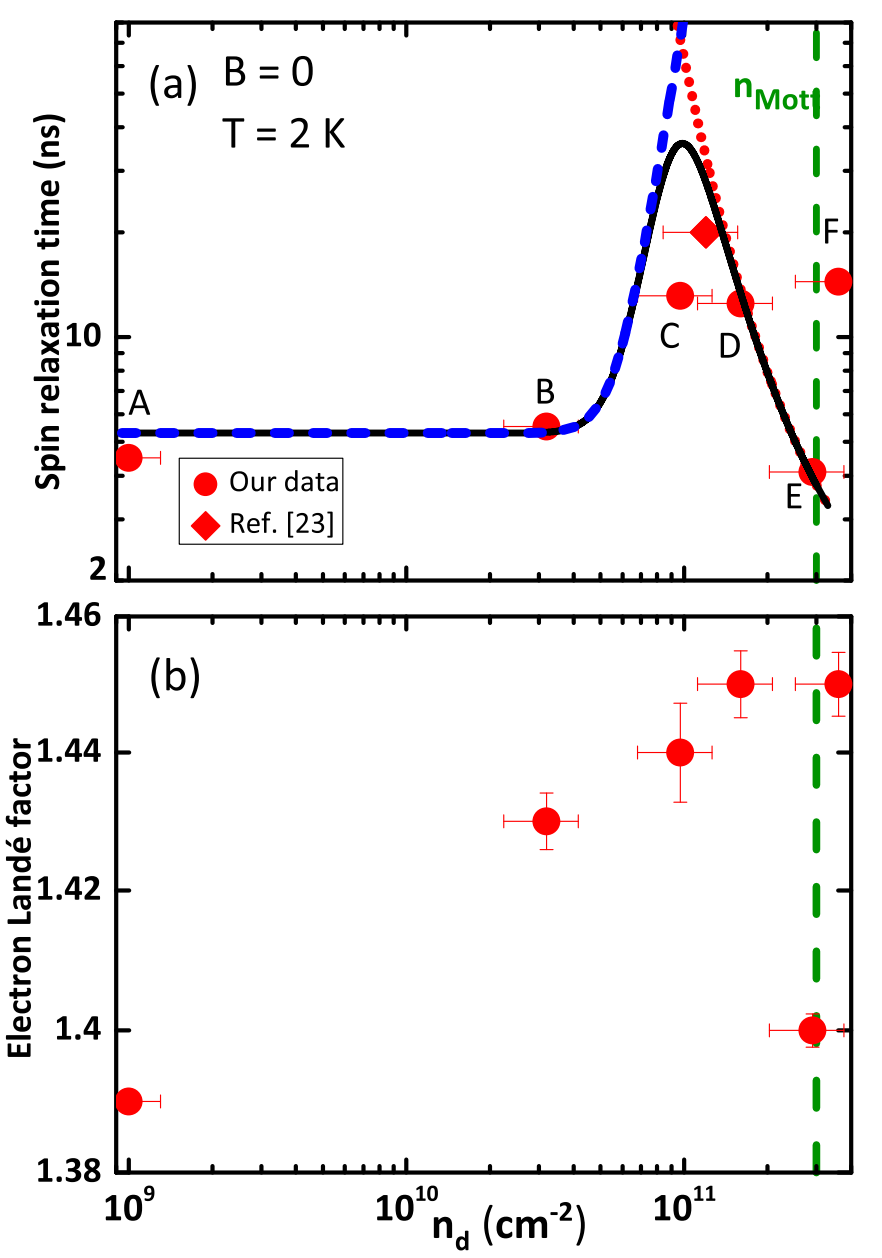

FIG. 5. (a) Measured spin relaxation times in samples A-F (full disks); the full diamond represents a measurement of Ref. [23]. The black solid line represents the spin relaxation time as a function of the doping concentration according to Eq. (12). The relaxation mechanisms for electrons bound to donors in the insulating regime are also represented: hyperfine interaction, $\tau_{s n}+T_{\Delta}^{e}$ [see Eq. (12)] (dashed line), and anisotropic exchange interaction, Eq. (10) (dotted line). (b) Transverse electron Landé factor $g_{e}^{\perp}$ as a function of the doping concentration (full disks).

\section{DISCUSSION}

For bulk GaAs in the insulating regime and at low temperature $[7,19]$, the dependence of the spin relaxation time on the doping concentration was explained as a result of two main relaxation mechanisms: the hyperfine interaction and the anisotropic exchange interaction. In this section, we adapt the theory developed for bulk crystals to a QW structure.

At low doping concentration, the donors are isolated and the electron spin relaxation via hyperfine interaction with the nuclei is the dominant relaxation mechanism. It was shown that for an ensemble of localized electrons, the spin polarization firstly drops down to $10 \%$ of its initial value within a characteristic time $2 T_{\Delta}^{e}$ and afterwards, the electronic spin polarization reaches a constant value of $1 / 3$ of its initial value, keeping it during a time of the order of a microsecond
(Ref. [29]). $T_{\Delta}^{e}$ is written as [30]

$$
T_{\Delta}^{e}=\hbar \sqrt{\frac{3 N_{L}}{2 n \sum_{i} I_{i}\left(I_{i}+1\right)\left(A_{i}\right)^{2} P_{i}}},
$$

where $A_{i}$ are the hyperfine constants, $I_{i}$ are the nuclear spin quantum numbers, $P_{i}$ the abundance of the nuclear species, and $N_{L}$ is the number of nuclei within the wave-function envelope of an electron. The sum runs over all nuclei of the crystal basis and for each nucleus over its nonvanishing nuclear spin. For CdTe, a unit cell contains one $\mathrm{Cd}$ and one Te atom. The stable isotopes of cadmium and tellurium with nonzero nuclear spin are $\mathrm{Cd}^{111}, \mathrm{Cd}^{113}, \mathrm{Te}^{123}$, and $\mathrm{Te}^{125}$; they all have a nuclear spin $I=1 / 2$. Both $\mathrm{Cd}$ isotopes have almost the same hyperfine constant, $A_{\mathrm{Cd}}=31 \mu \mathrm{eV}$, and they have together a natural abundance of $P_{\mathrm{Cd}}=25 \%$. The hyperfine constant of $\mathrm{Te}^{123}$ and $\mathrm{Te}^{125}$ is $A_{\mathrm{Te}}=-45 \mu \mathrm{eV}$, and they have an abundance of $P_{\mathrm{Te}}=8 \%$. To calculate $N_{L}=2 V_{L} / \Omega$ with $\Omega$ being the volume of a unit cell and $V_{L}$ being the effective volume defined as $\left[\int|\Psi(\vec{r})|^{4} d \vec{r}\right]^{-1}[29]$, we take $\Psi(\vec{r})$ as a modified hydrogenic envelope wave function for the electron in a finite QW of thickness $L$ [see the Appendix]. We took an effective mass $m^{*}=0.11 m_{0}$ (with $m_{0}$, the free electron mass) [31] and a dielectric constant of $\varepsilon_{r}=10.2$ [32]. Then, a variational calculation allows us to obtain for $L=8 \mathrm{~nm}$, $T_{\Delta}^{e}=5.6 \mathrm{~ns}$, with a barrier $\mathrm{Mg}$ concentration $x_{\mathrm{Mg}}=11 \%$. (Note that for sample $\mathrm{A}, x_{\mathrm{Mg}}=18 \%$, leading to a higher QW confinement and $\left.T_{\Delta}^{e}=5.3 \mathrm{~ns}\right)$. We emphasize that the experimental value for $T_{2}(0)$ obtained in sample $\mathrm{A}$ is very close to this estimation: the spin relaxation time for sample A is mostly limited by the hyperfine interaction.

For intermediate doping concentrations (samples B and $\mathrm{C}$ in Fig. 5), the main electronic spin relaxation mechanism is associated with the tunnel jumps of the electronic spin and with the hyperfine interaction. Each donor site has its own nuclear environment and each set of nuclear spins in the vicinity of a donor corresponds to an average nuclear field, i.e., an effective local magnetic field whose orientation and intensity depend on the considered donor site. The electron spin undergoes, at a given site, a rotation around the effective hyperfine field during the correlation time $\tau_{c}$. Then, the electronic spin is rotated around a different axis after each jump, and the spin relaxation rate in this regime is given by [33]

$$
\frac{1}{\tau_{s n}}=\frac{3}{2} \frac{\tau_{c}}{\left(T_{\Delta}^{e}\right)^{2}},
$$

where $\tau_{c}$ has been estimated as a function of the exchange constant $J(r)$ [7]:

$$
\tau_{c} \approx \frac{\hbar}{\xi J\left(r_{c}\right)}
$$

with $r_{c}$ the average characteristic distance between interacting donors: $r_{\mathrm{c}}=b\left(n_{2 \mathrm{D}}\right)^{-1 / 2}$ or $r_{\mathrm{c}}=b\left(n_{3 \mathrm{D}}\right)^{-1 / 3}$ in $2 \mathrm{D}$ and $3 \mathrm{D}$ respectively, and $b$ and $\xi$ are numerical factors of the order of unity. $b$ is expected to be between 0.54 and 0.8 , and a value of $\xi=0.8$ has been taken in bulk GaAs performing the fit of the experimental data [7]. 
In our case, we consider the exchange energy $2 J(r)$ of a pair of donors inside an infinite $\mathrm{QW}$ of thickness $L$ [34]:

$$
2 J(r)=2 J_{0} E_{\mathrm{h}}^{*}\left[1+(A R)^{2}\right]^{\frac{\beta}{2}} \exp [-\gamma R-\beta A R \operatorname{arctg}(A R)],
$$

with $R=r / a_{B}^{*}, r$ is the distance between donors, $a_{B}^{*}=$ $4.91 \mathrm{~nm}$ is the bulk Bohr radius, $E_{h}^{*}=29 \mathrm{meV}$ is the Hartree energy, and $2 J_{0}=1.34$ is the splitting energy in $E_{h}^{*}$ units between the ground state and the first excited state of a helium atom in a $\mathrm{QW}$ of thickness $L=8 \mathrm{~nm}$. The parameters $A=0.71, \beta=1.8$, and $\gamma=0.23$ are extracted from an interpolation method between $2 J_{0}$ and the exchange energy at large distances between the donors in an infinite QW [34]. We are confident that taking an infinite $\mathrm{QW}$ to calculate $2 J(r)$ is a reasonable approximation for our CdTe QWs of 8-nm thickness. Indeed, this thickness implies that the barriers strongly confine the wave function of a donor-bound electron centered in the QW, because of the value $a_{B}^{*}=4.91 \mathrm{~nm}$ of the bulk Bohr radius. This wave function is quasi-entirely located inside the QW, with only a small portion overflowing in the barrier, and it should then possess a shape very similar to the one of the wave function in an infinite QW; a mark of this fact comes from the value of the Bohr radius in the QW center plane for finite barriers, $4.5 \mathrm{~nm}$ (see Appendix), which is very close to the one calculated in an infinite QW, $4.4 \mathrm{~nm}$ [34]. As the exchange energy $2 J(r)$ depends on the overlap of electron wave functions for two donors separated by a distance $r$, our calculated $2 J(r)$ in an infinite QW is expected to be an acceptable approximation of the exact one.

As the concentration is increased to approach the metalinsulator transition (samples D and E), a second mechanism of spin relaxation becomes important. This mechanism is due to the anisotropic exchange interaction between two neighboring donors. During each exchange interaction, the spin-orbit interaction generates a rotation of an angle $\theta$, simultaneous and in the opposite direction, of each of the two electron spins that exchange their positions on the donors. To each pair of donors corresponds an axis and an angle of rotation. The expression for the ensemble spin relaxation time due to this mechanism is given by [7]

$$
\frac{1}{\tau_{s a}}=\frac{2}{3} \frac{\theta^{2}}{\tau_{c}}
$$

In semiconductor structures (QWs or quantum dots), $\theta$ is written as follows [35]:

$$
\theta=\frac{2 \alpha_{s o} \hbar}{\sqrt{2 m_{e}^{*} E_{g}}}\left\langle k_{z}^{2}\right\rangle r
$$

where $m_{e}^{*}$ is the effective electron mass, $E_{g}$ is the band gap energy, $r$ is the distance between the donors, $\alpha_{s o}$ is the spinorbit constant, and $\left\langle k_{z}^{2}\right\rangle$ is the average value of the squared $z$ component of the electron wave vector.

As we have already remarked, Fig. 5(a) shows that after the metal-insulator transition (sample F), there is an increase in the spin relaxation time. In this regime, the mechanism that governs the spin relaxation time at low temperature for electrons is the D'yakonov-Perel mechanism, the theory of which will be not discussed in this article.

The solid line in Fig. 5(a) represents a fit of the experimental data to the theoretical relaxation time of donor-bound elec- trons at low temperature, in the insulating regime, taking into account the hyperfine and anisotropic exchange interactions:

$$
\tau_{s}=\left(\frac{1}{\tau_{s n}+T_{\Delta}^{e}}+\frac{1}{\tau_{s a}}\right)^{-1},
$$

with $\tau_{s n}$ and $\tau_{s a}$ given by the expressions (7) and (10). The model (12) agrees with the experimental data using reasonable values of the parameters: $T_{\Delta}^{e}=5.3 \mathrm{~ns}, b=0.8, \xi=0.1$, and $\theta=(0.022 \pm 0.003) \frac{r}{a_{B}^{*}}$.

From the prefactor found in the expression of $\theta$, it is possible to deduce, using Eq. (11), a range of values for the spinorbit constant $\alpha_{s o}$ in CdTe. We obtain finally $\alpha_{s o}=0.079 \pm$ 0.011 , taking $E_{g}=1.606 \mathrm{eV}$ and $\left\langle k_{z}^{2}\right\rangle=6.1 \times 10^{-2} \mathrm{~nm}^{-2}$ calculated using a hydrogenic wave function inside of a finite QW of thickness $L=8 \mathrm{~nm}$ (see the Appendix). This value for $\alpha_{s o}$ is close to the one that can be extracted from the theoretical values reported in the literature for the spin splitting of the $\Gamma_{6}$ conduction band in CdTe: $\gamma_{c}=11.75 \mathrm{eV}^{3}$ [36] and $\gamma_{c}=8.5 \mathrm{eV} \AA^{3}$ [37]; using the relation [38]

$$
\alpha_{s o}=\frac{2 \gamma_{c}}{\hbar^{3}} \sqrt{2 m_{e}^{* 3} E_{g}}
$$

one obtains $\alpha_{s o}=0.073$ and $\alpha_{s o}=0.053$, respectively.

Now, we use our experimental value of the spin-orbit constant, $\alpha_{s o}=0.079$, to plot in Fig. 6(a) the predicted spin relaxation time in bulk CdTe [solid line in Fig. 6(a)], and make a comparison with the available experimental values. We have used Eq. (12) and the corresponding expressions for $\theta$ and $J$ in 3D [34,39], taking for the parameters $b$ and $\xi$ the values 0.7 and 0.8 respectively, which are in the range proposed by Ref. [7]. The dephasing time $T_{\Delta}^{e}$ in bulk is calculated following Eq. (6) (see Ref. [40]).

We note first that the theoretical spin relaxation times in bulk CdTe are longer than the relaxation times measured in a 8-nm CdTe QW. In general, in the low-doping regime (large $R), T_{\Delta}^{e}$ in a QW is slightly smaller than in bulk, since the localization of the electron wave function is increased. Also, the electron exchange energy, $2 J$, is smaller [see Fig. 7(a) of Ref. [34]], therefore a shorter spin relaxation time in this regime is expected for a QW. At high doping concentrations (short $R$ ), a similar analysis leads us to conclude that the expected relaxation time is also shorter in a $\mathrm{QW}\left(J_{3 \mathrm{D}}\right.$ and $\theta_{3 \mathrm{D}}$ are smaller than $J_{\mathrm{QW}}, \theta_{\mathrm{QW}}$, therefore $\left.1 / \tau_{s a, 3 \mathrm{D}}<1 / \tau_{s a, Q W}\right)$. Thus, we can expect that the spin relaxation time in bulk is always longer than in a QW.

We also note that, in bulk, the reported experimental values shown in Fig. 6(a) are very small compared to the ones predicted by the theory. In particular, for very small doping concentrations $\left(<10^{15} \mathrm{~cm}^{-3}\right)$ the estimated spin relaxation time due the hyperfine interaction is of the order of $10 \mathrm{~ns}$ and the measured one is more than two orders of magnitude smaller. A similar situation is observed for the maximum relaxation time, which is the result of a combination of the hyperfine interaction and anisotropic exchange interaction: the theoretical value is of the order of $100 \mathrm{~ns}$ and the experimental reported value is equal to $2 \mathrm{~ns}$. Moreover, the maximum of the experimental curve occurs at a higher doping concentration than the calculated one. The presence of compensation sites in the sample, which reduces the nominal doping concentration 

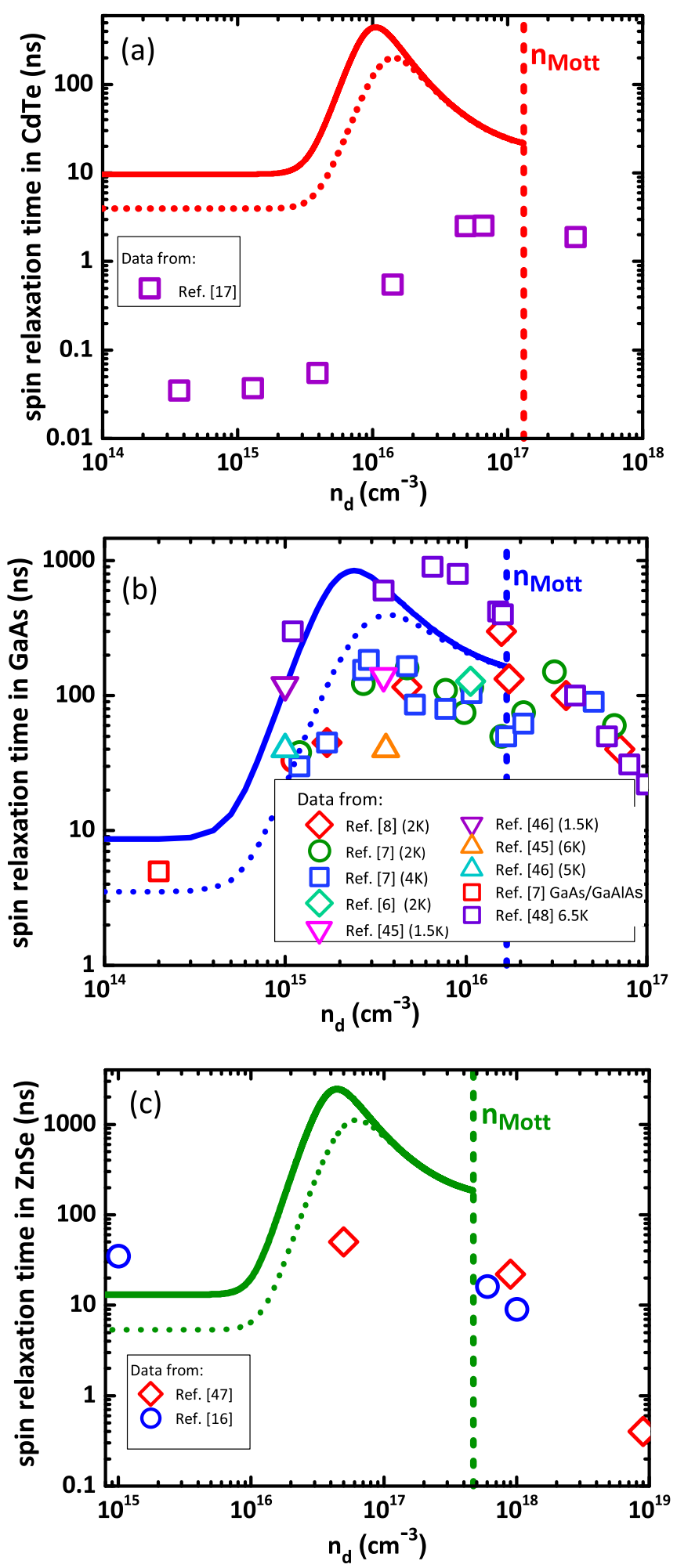

FIG. 6. Theoretical spin relaxation times (solid lines) as a function of the doping concentration for (a) CdTe bulk, (b) GaAs bulk, and (c) $\mathrm{ZnSe}$ bulk. The dotted lines represent the spin relaxation times when a volume of a sphere of radius $a_{B}^{*}$ is considered as effective volume $V_{L}$ in the calculation of $T_{\Delta}^{e}$ (see Sec. IV). We have also reported different experimental results concerning spin relaxation times at low temperature for each material (see Refs. [45-48]). could be at the origin of this last disagreement. Regarding the magnitude of the disagreements, they are likely related to the conditions of the concrete experimental measurements. Sprinzl et al. [17] obtained the spin relaxation time by using pump-probe experiments similar to our experiments, but they used femtosecond pulses tuned to the band gap of CdTe. Instead, in our experiments, we use picosecond pulses tuned to the $D^{0} X$ transition. A femtosecond pulse tuned to the bandgap is able to create a population of delocalized electrons in the conduction band that can remain even after recombination of holes with localized electrons and also can produce a heat-up of localized electrons. In our experiments, we have performed a resonant excitation of $D^{0} X$ transition to minimize all these effects, because they introduce additional spin relaxation mechanisms [19]. Moreover, the concentration of photoexcited carriers reported in Ref. [17], $N_{e x}=5 \times$ $10^{16} \mathrm{~cm}^{-3}$, is comparable to the concentration range of the studied samples (and even two orders of magnitude larger than the lower concentration). A significant shortening of the spin relaxation time is then expected, as it has been observed in Ref. [6] for high photoexcited carrier concentrations $N_{e x}$ (close to the nominal concentration $n$ ).

Now, let us compare bulk CdTe spin relaxation times with those theoretically estimated and experimentally observed in bulk GaAs [Fig. 6(b)] and ZnSe [Fig. 6(c)] materials. As we have done for bulk CdTe, we have used Eq. (12) to calculate the spin relaxation time. The parameters involved in the calculation of $T_{\Delta}^{e}$ for each material are reported in Table I. The value for the spin-orbit constant of $\mathrm{ZnSe}$ has been calculated using $\gamma_{c}=1.62 \mathrm{eV} \cdot \AA^{3}$ [36] and Eq. (13).

We see in Fig. 6(b) that the maximum values for the spin relaxation time predicted for bulk GaAs are longer than those predicted for bulk CdTe, and appear at lower doping concentrations. This is explained as follows. First, at low doping concentrations (large $R$ ), the exchange energy is smaller in CdTe than in GaAs [see Fig. 7(b) of Ref. [34]], therefore $\tau_{s n}$, which is directly proportional to $J$, is smaller in CdTe. Second, for high doping concentrations (short $R$ ) [see Fig. 7(a) of Ref. [34]], $J$ and $\theta$ are greater for CdTe than for GaAs (due to the greater value of the spin-orbit constant and smaller Bohr radius in $\mathrm{CdTe}$ ), therefore $\tau_{s a}$, which is inversely proportional to them, is smaller. The metal-insulator transition appears for GaAs at a lower doping concentration than for CdTe, since the Bohr radius in CdTe is smaller than in GaAs.

The experimental results for GaAs are slightly shorter than those predicted by the theory but the agreement is rather good. Mostly these have been obtained by Hanle depolarization of photoluminescence, resonant pump-probe experiments at the $D^{0} X$ transition with picosecond pulses or time resolved polarization spectroscopy. In bulk materials, a circularly polarized excitation creates heavy and light excitons, and then additional spin relaxation mechanisms can appear during the exciton lifetime, affecting also the spin relaxation of the localized electrons. In Fig. 6, the dotted lines represent the theoretical predictions obtained by imposing the volume of a sphere of radius equal to the effective bulk Bohr radius, $V_{L}=$ $4 \pi a_{B}^{* 3} / 3$, for the calculation of $T_{\Delta}^{e}$, as is done in Refs. $[23,16]$. 
TABLE I. Parameters required for the calculation of the spin dephasing time $T_{\Delta}^{e}$ due to the hyperfine interaction in GaAs, CdTe, and ZnSe.

\begin{tabular}{|c|c|c|c|c|c|c|c|c|}
\hline \multirow[t]{2}{*}{ GaAs } & \multirow[t]{2}{*}{$0.067^{\mathrm{a}}$} & \multirow[t]{2}{*}{$12.35^{\mathrm{b}}$} & $\mathrm{Ga}^{71}$ & $I_{\mathrm{Ga}}=3 / 2$ & $P_{\mathrm{Ga}}=100 \%$ & $\sum_{\mathrm{I}}\left(A^{\mathrm{i}}\right)^{2}$ & \multirow{2}{*}{$0.067^{\mathrm{d}}$} & \multirow{2}{*}{8.6} \\
\hline & & & $\mathrm{As}^{75}$ & $I_{\mathrm{As}}=3 / 2$ & $P_{\mathrm{As}}=100 \%$ & $=1.210^{-3} \mathrm{meV}^{2 \mathrm{c}}$ & & \\
\hline \multirow{2}{*}{ CdTe } & \multirow{2}{*}{$0.11^{\mathrm{e}}$} & \multirow{2}{*}{$10.2^{\mathrm{f}}$} & $\mathrm{Te}^{123}$ & $I_{\mathrm{Te}}=1 / 2$ & $P_{\mathrm{Te}}=8 \%$ & $A^{\mathrm{cd}}=31 \mu \mathrm{eV}$ & \multirow{2}{*}{0.079} & \multirow{2}{*}{9.7} \\
\hline & & & $\mathrm{Te}^{125}$ & & & & & \\
\hline \multirow{2}{*}{$\mathrm{ZnSe}$} & \multirow{2}{*}{$0.145^{\mathrm{g}}$} & \multirow{2}{*}{$8.8^{\mathrm{b}}$} & $\mathrm{Zn}^{67}$ & $I_{\mathrm{Zn}}=5 / 2$ & $P_{\mathrm{Zn}}=4.11 \%$ & $A^{\mathrm{Zn}}=3.7 \mu \mathrm{eV}$ & \multirow{2}{*}{0.021} & \multirow{2}{*}{13.1} \\
\hline & & & $\mathrm{Se}^{77}$ & $I_{\mathrm{Se}}=1 / 2$ & $P_{\mathrm{Se}}=7.58 \%$ & $A^{\mathrm{Se}}=33.6 \mu \mathrm{eV}$ & & \\
\hline
\end{tabular}

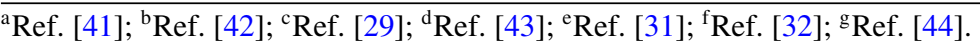

Figure 6(c) shows that the predicted maximum spin relaxation time for $\mathrm{ZnSe}$ is of the order of $1 \mu \mathrm{s}$. Recent studies have shown spin relaxation times around $30 \mathrm{~ns}$ at low doping concentrations [16], but there are no other studies in the insulating regime. Considering the same theoretical model for the spin relaxation time in CdTe and GaAs, $\mathrm{ZnSe}$ appears as one possible candidate for obtaining long spin relaxation times.

\section{CONCLUSION}

The insertion of a neutral iodine donor in a QW leads to a system that increases the localization of the electron wave function with respect to the $3 \mathrm{D}$ material. It allows also a higher efficiency of the orientation of the electronic spin by using optical excitations. By using picosecond pulses in PFR experiments and a resonant excitation, we find that the spin relaxation time dependence on the doping concentration at low temperature follows a nonmonotonous behavior in the insulator regime, similar to the one observed in the most studied semiconductor material, GaAs. This behavior has been successfully explained by an interplay of two main spin relaxation mechanisms: hyperfine interaction and anisotropic exchange interaction. By adapting existing 3D models to a QW, we have obtained the value of the CdTe spin-orbit constant: $\alpha_{s o}=0.079$. Using this value, we estimated the spin relaxation time for bulk CdTe and compared it with GaAs, and with $\mathrm{ZnSe}$, a promising II-VI material, which appears as one possible candidate for obtaining long spin relaxation times, according to the spin relaxation mechanisms considered here.

\section{APPENDIX: DETERMINATION OF THE BINDING ENERGY, BOHR RADIUS, AND $\left\langle k_{z}^{2}\right\rangle$ TERM FOR A DONOR BOUND ELECTRON INSIDE OF A FINITE QUANTUM WELL}

The Hamiltonian describing an electron bound to a donor inside of a finite $\mathrm{QW}$ of thickness $L$ in dimensional units is written as

$$
H(z)=-\frac{\hbar^{2}}{2 m_{e}^{*}} \Delta-\frac{e^{2}}{4 \pi \varepsilon_{0} \varepsilon_{r}} \frac{1}{r}+V(z)
$$

where $m_{e}^{*}$ is the electron effective mass, $V(z)$ is the $\mathrm{QW}$ confinement potential defined by $V(z)=0$ for $|z| \leqslant \frac{L}{2}$ and $V(z)=V_{0}$ for $|z|>\frac{L}{2}, \Delta$ is the Laplacian operator, and $r$ is the distance of the electron to the origin of the coordinate system.

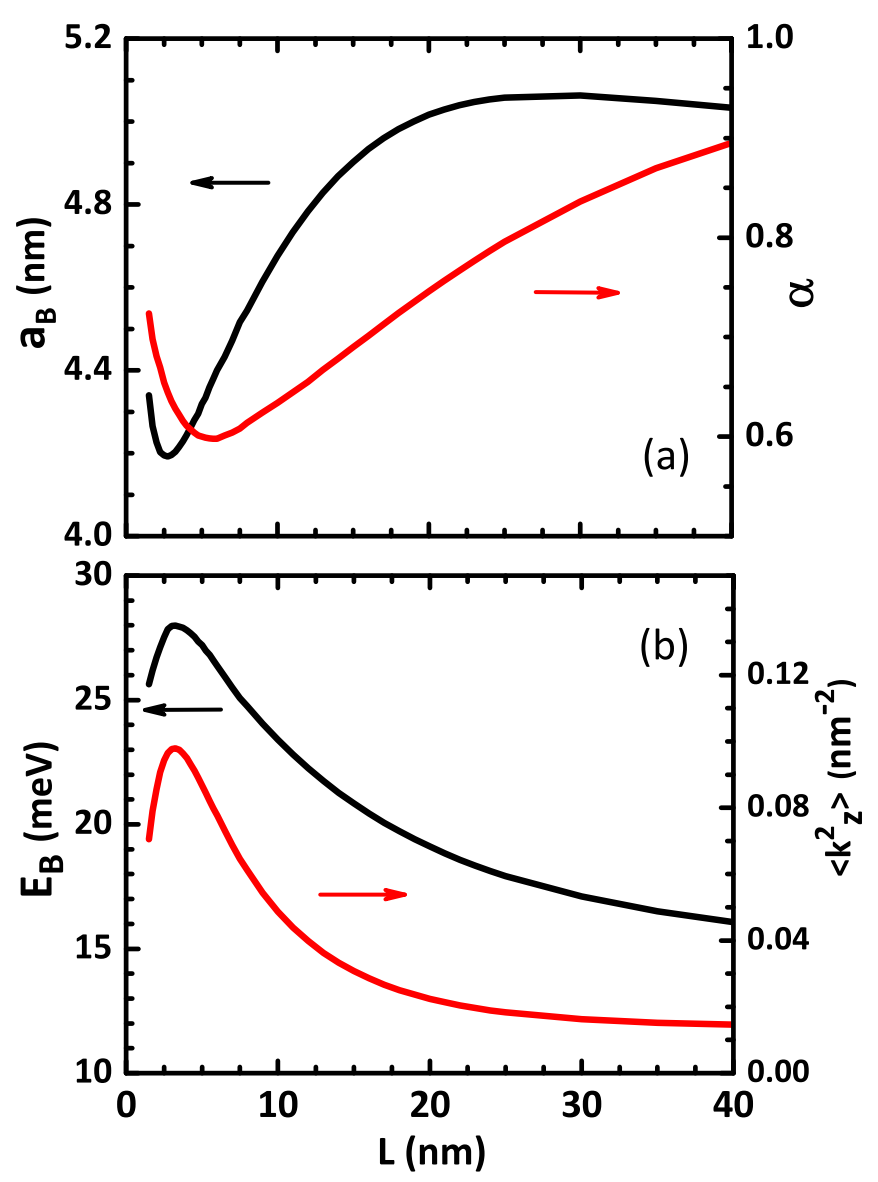

FIG. 7. (a) Bohr radius $a_{B}$ (left axis) and variational parameter $\alpha$ (right axis) of an electron bound to a donor in the middle of a finite QW of thickness $L$. (b) Binding energy $E_{B}$ (left axis) and $\left\langle k_{z}^{2}\right\rangle$ (right axis) for different thicknesses $L$ of a finite QW with a barrier height of $V_{0}=125 \mathrm{meV}$. 
The electron binding energy $E_{B}(L)$ and the Bohr radius $a_{B}(L)$ are determined by means of the variational method. The trial wave function is taken in the following form:

$$
\Psi(\vec{r})=B \chi(z) e^{-\frac{\sqrt{\rho^{2}+\alpha^{2} z^{2}}}{a_{B}}},
$$

where $\chi(z)$ is the QW envelope wave function, $B$ is the normalization coefficient, and $a_{B}$ and $\alpha$ are the variational parameters.

First, we have solved the equation associated with the envelope wave function in the QW:

$$
H_{z} \chi(z)=E_{0} \chi(z)
$$

with $H_{z}=-\frac{\hbar^{2}}{2 m} \frac{d^{2}}{d z^{2}}+V(z)$ and $E_{0}$ being the QW lowest energy confinement. We have then minimized the mean energy $\langle\Psi|H| \Psi\rangle$ to obtain the binding energy defined by $E_{B}=E_{0}-$ $\langle\Psi|H| \Psi\rangle$ and the effective Bohr radius $a_{B}$, for a given QW thickness $L$. Figure 7(a) shows the $L$ dependence of the variational parameters, $\alpha$ and $a_{B}$, for a barrier height $V_{0}=$
$125 \mathrm{meV}$ (corresponding to a conduction band offset of $70 \%$ and a barrier $\mathrm{Mg}$ concentration of $11 \%$ ). At large $\mathrm{QW}$ width, one tends to the 3D limit, with $\alpha=1$ and $a_{B}=a_{B}^{3 \mathrm{D}}$. When $L$ decreases, one has a commonly observed behavior: both parameters decrease, then increase when $L$ crosses a value close to the Bohr radius. It is well known that for a finite barrier, when the QW width decreases, the wave function starts to get more confined, before spreading in the barrier for $L$ smaller than the Bohr radius. The $\alpha$ and $a_{B}$ behaviors are the signature of this effect, as can be seen for $L<5 \mathrm{~nm}$ in Fig. 7(a).

Using the expression (A2) for the wave function, the coefficient $\left\langle k_{z}^{2}\right\rangle=\left\langle\Psi\left|\left(-i \frac{d}{d z}\right)^{2}\right| \Psi\right\rangle$ is also calculated. Figure 7(b) shows this coefficient $\left\langle k_{z}^{2}\right\rangle$ and the binding energy $E_{B}$ versus the QW width $L$. Starting from the bulk binding energy, for large $L$ values, one observes a maximum binding energy $E_{B}=$ $2 E_{B}^{3 \mathrm{D}}$ for $L \approx a_{B}$. A similar behavior is shown for $\left\langle k_{z}^{2}\right\rangle$. For a thickness $L=8 \mathrm{~nm}$, one has $E_{B}=24.75 \mathrm{meV}$ and $\left\langle k_{z}^{2}\right\rangle=$ $6.1 \times 10^{-2} \mathrm{~nm}^{-2}$. One can estimate $V_{L}=1.0 \times 10^{3} \mathrm{~nm}^{3}$ [29], leading to $T_{\Delta}=5.6 \mathrm{~ns}$.
[1] T. D. Ladd, F. Jelezko, R. Laflamme, Y. Nakamura, C. Monroe, and J. L. O'Brien, Nature (London) 464, 45 (2010).

[2] K. Heshami, D. G. England, P. C. Humphreys, P. J. Bustard, V. M. Acosta, J. Nunn, and B. J. Sussman, J. Mod. Opt. 63, 2005 (2016).

[3] K. Saeedi, S. Simmons, J. Z. Salvail, P. Dluhy, H. Riemann, N. V. Abrosimov, P. Becker, H. J. Pohl, J. J. Morton, and M. L. Thewalt, Science 342, 830 (2013).

[4] T. F. Watson, B. Weber, Y. L. Hsueh, L. C. L. Hollenberg, R. Rahman, and M. Y. Simmons, Sci. Adv. 3, e1602811 (2017).

[5] C. Weisbuch, Ph.D. thesis, Paris-VII University, 1977.

[6] J. M. Kikkawa and D. D. Awschalom, Phys. Rev. Lett. 80, 4313 (1998).

[7] R. I. Dzhioev, K. V. Kavokin, V. L. Korenev, M. V. Lazarev, B. Ya. Meltser, M. N. Stepanova, B. P. Zakharchenya, D. Gammon, and D. S. Katzer, Phys. Rev. B 66, 245204 (2002).

[8] V. V. Belykh, K.V. Kavokin, D. R. Yakovlev, and M. Bayer, Phys. Rev. B 96, 241201(R) (2017).

[9] M. Römer, H. Bernien, G. Müller, D. Schuh, J. Hübner, and M. Oestreich, Phys. Rev. B 81, 075216 (2010).

[10] S. A. Crooker, L. Cheng, and D. L. Smith, Phys. Rev. B 79, 035208 (2009).

[11] S. Ghosh, V. Sih, W. H. Lau, D. D. Awschalom, S.-Y. Bae, S. Wang, S. Vaidya, and G. Chapline, Appl. Phys. Lett. 86, 232507 (2005).

[12] D. Guzun, E. A. DeCuir Jr., Vas. P. Kunets, Yu. I. Mazur, G. J. Salamo, S. Q. Murphy, P. A. R. Dilhani Jayathilaka, T. D. Mishima, and M. B. Santos, Appl. Phys. Lett. 95, 241903 (2009).

[13] B. N. Murdin et al., Phys. Rev. B 72, 085346 (2005).

[14] B. Beschoten, E. Johnston-Halperin, D. K. Young, M. Poggio, J. E. Grimaldi, S. Keller, S. P. DenBaars, U. K. Mishra, E. L. Hu, and D. D. Awschalom, Phys. Rev. B 63, 121202(R) (2001).

[15] J. H. Buss, J. Rudolph, S. Shvarkov, H. Hardtdegen, A. D. Wiek, and D. Hagele, Appl. Phys. Lett. 102, 192102 (2013).
[16] A. Greilich, A. Pawlis, F. Liu, O.A. Yugov, D. R. Yakovlev, K. Lischka, Y. Yamamoto, and M. Bayer, Phys. Rev. B 85, 121303(R) (2012).

[17] D. Sprinzl, P. Horodyská, N. Tesařová, E. Rozkotová, E. Belas, R. Grill, P. Malý, and P. Němec, Phys. Rev. B 82, 153201 (2010).

[18] K.V. Kavokin, Phys. Rev. B 64, 075305 (2001).

[19] K.V. Kavokin, Semicond. Sci. Technol. 23, 114009 (2008).

[20] P. I. Tamborenea, T. Wellens, D. Weinmann, and R. A. Jalabert, Phys. Rev. B 96, 125205 (2017).

[21] M. Chamarro, F. Bernardot, and C. Testelin, J. Phys. Condens. Matter 19, 445007 (2007).

[22] J. Tribollet, F. Bernardot, M. Menant, G. Karczewski, C. Testelin, and M. Chamarro, Phys. Rev. B 68, 235316 (2003).

[23] J. Tribollet, E. Aubry, G. Karczewski, B. Sermage, F. Bernardot, C. Testelin, and M. Chamarro, Phys. Rev. B 75, 205304 (2007).

[24] P. Harrison, S. J. Weston, T. Piorek, T. Stirner, W. E. Hagston, J. E. Nicholls, and M. O’Neill, Superlattices Microstruct. 14, 249 (1993).

[25] K. Kheng, R. T. Cox, Y. Merle d'Aubigné, Franck Bassani, K. Saminadayar, and S. Tatarenko, Phys. Rev. Lett. 71, 1752 (1993).

[26] P. Grinberg, F. Bernardot, B. Eble, G. Karczewski, C. Testelin, and M. Chamarro, J. Appl. Phys. 119, 123906 (2016).

[27] A. A. Sirenko, T. Ruf, M. Cardona, D. R. Yakovlev, W. Ossau, A. Waag, and G. Landwehr, Phys. Rev. B 56, 2114 (1997).

[28] N. F. Mott, Metal-Insulator Transitions (Taylor and Francis, London, 1990).

[29] I. A. Merkulov, Al. L. Efros, and M. Rosen, Phys. Rev. B 65, 205309 (2002).

[30] There are misprints in Ref. [29]: in Eq. (8), the factor 8 must be changed to $n=2$; and in Eqs. (7) and (11), the factor 16 must be changed to $2 n=4$. 
[31] D.T. F. Marple, Phys. Rev. 129, 2466 (1963).

[32] S. Perkowitz and R. H. Thorland, Phys. Rev. B 9, 545 (1974).

[33] M. I. D'yakonov and V. I. Perel, Zh. Eksp. Teor. Fiz. 65, 362 (1973). [Sov. Phys. JETP 38, 177 (1974)].

[34] G. Garcia-Arellano, F. Bernardot, C. Testelin, and M. Chamarro, Phys. Rev. B 98, 195308 (2018).

[35] K. V. Kavokin, Phys. Rev. B 69, 075302 (2004).

[36] M. Cardona, N. E. Christensen, and G. Fasol, Phys. Rev. B 38, 1806 (1988).

[37] A. N. Chantis, M. van Schilfgaarde, and T. Kotani, Phys. Rev. Lett. 96, 086405 (2006).

[38] G. E. Pikus and A. N. Titkov, in Optical Orientation, edited by F. Meier and B. P. Zakharchenya (North Holland, Amsterdam, 1984).

[39] L. P. Gor'kov and P. L. Krotkov, Phys. Rev. B 67, 033203 (2003).

[40] The dephasing time due to the hyperfine interaction $T_{\Delta}^{e}$ in bulk is calculated following Eq. (6), using a hydrogenic wave function $\Psi(\vec{r})=\frac{1}{\sqrt{\pi} a_{B}^{3 / 2}} e^{-r / a_{B}}$ for the effective volume $V_{L}=$ $\left[\int|\Psi(\vec{r})|^{4} d \vec{r}\right]^{-1}$ giving $V_{L}=8 \pi a_{B}^{* 3}$ (solid lines in Fig. 6). A better agreement with the experimental results is achieved using the volume of a sphere of radius equal to the effective bulk
Bohr radius $V_{L}=4 \pi a_{B}^{* 3} / 3$ (dashed lines in Fig. 6). Due to the quadratic dependence on $T_{\Delta}^{e}$ of $\tau_{s n}$, the difference of approximately $80 \%$ between the square of the two calculated values for $T_{\Delta}^{e}$ in GaAs and CdTe, produces a considerable difference for the maximum spin relaxation time predicted by the theory.

[41] W. Nakwaski, Physica B 210, 1 (1995).

[42] I. Strzalkowski, S. Joshi, and C. R. Crowell, Appl. Phys. Lett. 28, 350 (1976).

[43] B. Jusserand, D. Richards, G. Allan, C. Priester, and B. Etienne, Phys. Rev. B 51, 4707 (1995).

[44] I. Hernández-Calderón, in II-VI Semiconductor Materials and their Applications, edited by M. C. Tamargo (Taylor and Francis, New York, 2002).

[45] J. S. Colton, T. A. Kennedy, A. S. Bracker, and D. Gammon, Phys. Rev. B 69, 121307(R) (2004).

[46] J. S. Colton, M. E. Heeb, P. Schroeder, A. Stokes, L. R. Wienkes, and A. S. Bracker, Phys. Rev. B 75, 205201 (2007).

[47] I. Malajovich, J. M. Kikkawa, D. D. Awschalom, J. J Berry, and N. Samarth, J. Appl. Phys. 87, 5073 (2000).

[48] J. G. Lonnemann, E. P. Rugeramigabo, M. Oestreich, and J. Hübner, Phys. Rev. B 96, 045201 (2017). 